\title{
Coexistence of paraelectric/proton-glass and ferroelectric (antiferroelectric) orders in $\mathrm{Rb}_{1-x}\left(\mathrm{NH}_{4}\right)_{x} \mathrm{H}_{2} \mathrm{AsO}_{4}$ crystals
}

\author{
Z.Trybuła, J.Stankowski \\ Institute of Molecular Physics, Polish Academy of Sciences, \\ Smoluchowskiego 17, 60-179 Poznañ, Poland
}

Received June 17, 1998

This paper reviews the results of dielectric studies of the proton glass state in a mixed crystal Rubidium Ammonium Dihydrogen Arsenate (RADA) The coexistence of paraelectric/proton glass and ferroelectric or antiferroelectric orders, confirmed by other studies, has been discussed in detail. The phase diagram of RADA is asymmetric. The proton glass state exists for ammonium concentration in the range of $0.1<x<0.5$. The phase diagram of deuterated DRADA is presented. The proton glass state region in DRADA, $0.2<x<0.35$ is narrower than that for non-deuterated RADA. The effects of hydrostatic pressure on the dielectric properties in the proton glass state are presented. The glass temperature $T_{g}$ decreases with pressure and is expected to vanish at $5 \mathrm{kbar}$. Low temperature behaviour is still an open question, since there is no experimental evidence of $T_{g}(p)$ dependence below $4 \mathrm{~K}$ for proton glass systems.

Key words: domain structure, ferroelasticity

PACS: 77.22.ch, 77.22.gm, 64.70.-p, 74.84.-s

\section{Introduction}

The studies of the proton glass state in a mixed crystal of Rubidium Ammonium Dihydrogen Phosphate - $\mathrm{Rb}_{1-x}\left(\mathrm{NH}_{4}\right)_{x} \mathrm{H}_{2} \mathrm{PO}_{4}$ (RADP) were launched by Courtens [1] in 1982. At low ammonium concentration $x$ RADP has a distorted ferroelectric structure with a typical paraelectric-ferroelectric (P-FE) transition, while at high ammonium concentration ( $x$ close to 1 ) RADP exhibits a distorted paraelectric/aniferroelectric transition (P-AFE). Temperature of the phase transitions: $T_{c}$ of P-FE transition as well as $T_{N}$ of P-AFE transition significantly lowers with the increase of the concentration of the second component of a mixed crystal. For $\mathrm{NH}_{4}$ concentration $0.22 \leqslant x \leqslant 0.75$ [2], below the "freezing" temperature $T_{g}$, 
the competition between ferroelectric and antiferroelectric orderings leads to frustration of the protonic system and appearance of regions with a local short-range order but without any evidence of a long-range order (ferroelectric or antiferroelectric). The proton glass state was also detected in an isomorphous crystal of Rubidium Ammonium Dihydrogen Arsenate- $\mathrm{Rb}_{1-x}\left(\mathrm{NH}_{4}\right)_{x} \mathrm{H}_{2} \mathrm{AsO}_{4}$ (RADA) by Trybuła et al. [3] in the dielectric study. This paper will focus essentially on the properties of the RADA crystal. Earlier dielectric studies gave some insight into the properties of the proton glass state [4-7]. The phase diagram for RADA obtained by those studies is unlike that for RADP, and reveals the glass state existence in the range of $0.1 \leqslant x \leqslant 0.5[4,5]$.

Further studies showed [8-16] that the phase diagram is more complex, since paraelectric/proton-glass-ferroelectric or antiferroelectric phases coexist. This paper presents the up to date understanding of the proton-glass state. First, after a short introduction, dielectric results in the mixed RADA crystal will be reviewed. Then, the problem of phase coexistence in this crystal based on dielectric studies, discussion of the phase diagram and the effect of pressure and electric field on the proton glass state will be presented.

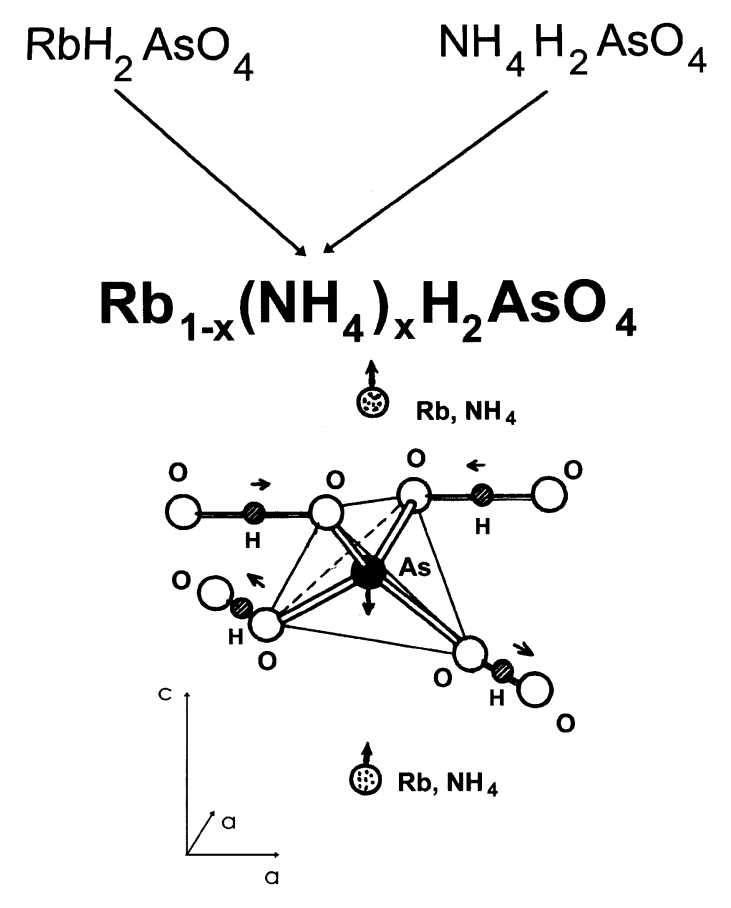

Figure 1. Ordered hydrogen-bonds system that gives spontaneous polarization.
Crystals of the $\mathrm{KH}_{2} \mathrm{PO}_{4}(\mathrm{KDP})$ family were the most extensively studied ferroelectrics $[17,18]$. Ferroelectric properties of these crystals originate from the ordering of the protons in hydrogen bonds linking tetrahedral PO4 or $\mathrm{AsO} 4$ units in the crystal structure. The unit cell is tetragonal. Spontaneous polarization is the result of proton ordering due to the shift along the c axis of the centre of $\mathrm{Rb}^{+}$or $\mathrm{NH}_{4}^{+}$ tetrahedrons with respect to phosphorus or arsenium atoms in $\mathrm{H}_{2} \mathrm{PO}_{4}$ or $\mathrm{H}_{2} \mathrm{AsO}_{4}$ groups, while hydrogen bonds are formed almost in (ab) plane (figure 1). In an ordered ferroelectric phase, below $T_{c}$, up-down configuration of hydrogen bonds (figure 3 ) is favoured. The change from up to down configuration leads to a switch in the polarization of the crystal. The next-lying excited state corresponds to lateral configurations - two protons are in a lateral position. These configurations are called Slater configurations [19]. Higher energies of the hydrogen bond network correspond to Takagi configurations [20] related to the ordering of a distorted tetrahedron with one, three, four protons or lack of protons at all (figure 2). Antiferroelectrically ordered ADP or ADA crystals, be- 
low Néel temperature $T_{N}$, have lateral configurations in the ground state. The first excited state corresponds to up- down configuration. In mixed crystals of RADP or RADA there is a small energy difference between the ground and excited states, so the multiplet ground state is possible, hence the proton glass state can occur (figure 3).

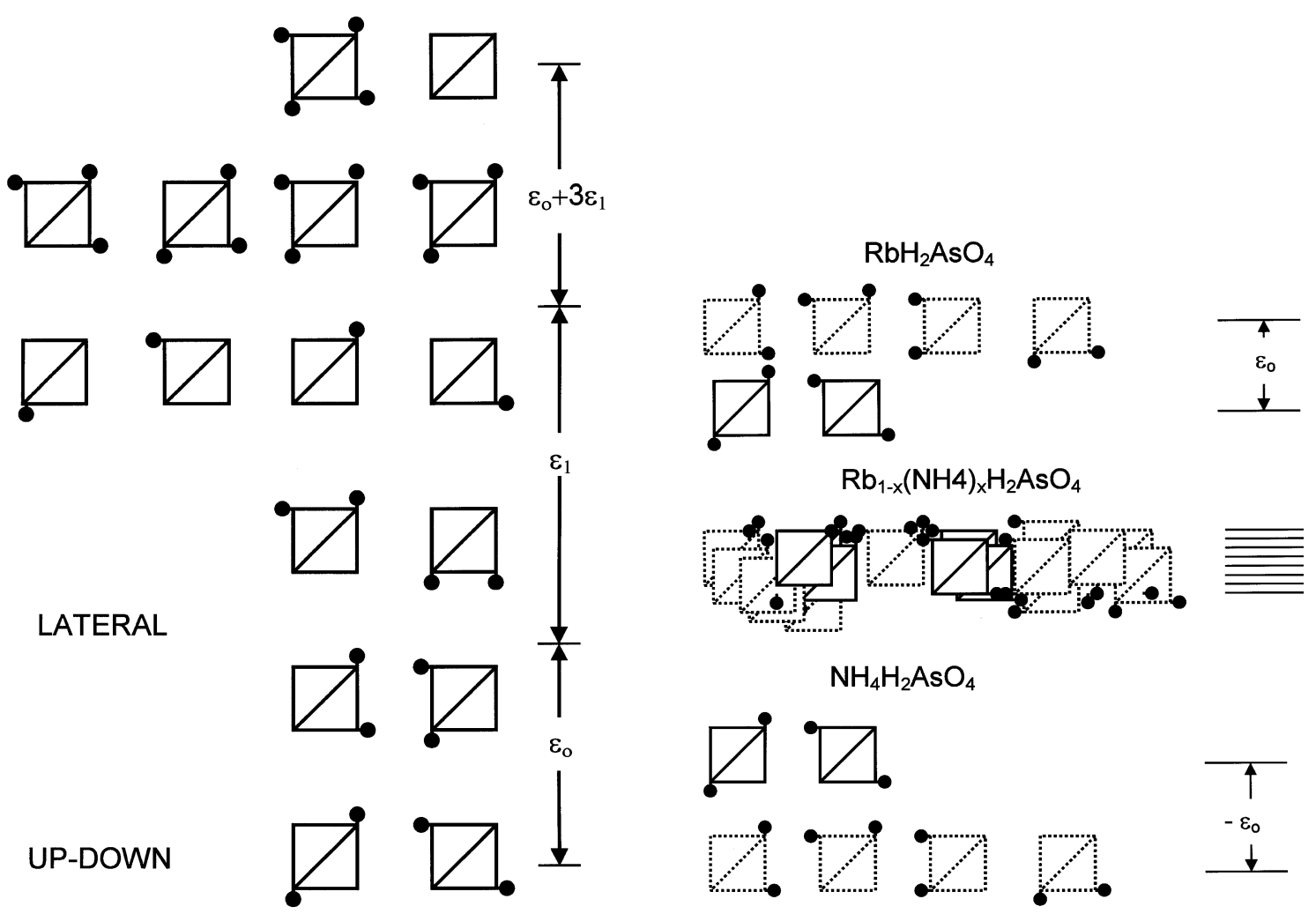

Figure 2. Configurational energies of 16 types of phosphate $\mathrm{PO}_{4}$ or arsenate $\mathrm{AsO}_{4}$ tetrahedra, represented in the pseudospin formalism and in terms of Slater energy $\varepsilon_{o}$ and Takagi energy $\varepsilon_{1}[52]$.
Figure 3. Proton configuration in ferroelectric RDA, proton glass RADA and antiferroelectric ADA.

\section{Review of dielectric studies of mixed RADA}

Since Courtens discovered the proton glass state in RADP [1-2], the temperature dependence of real $\varepsilon^{\prime}$ and imaginary $\varepsilon^{\prime \prime}$ parts of electric permittivity has been extensively studied [21-29]. We have contributed to this work with the observation of the proton glass state in a RADA mixed crystal [3]. First measurements were carried out in a microwave X-band range for measuring electric field frequency of $9.2 \mathrm{GHz}$. The methodology of $\varepsilon^{\prime}$ and $\varepsilon^{\prime \prime}$ measurement in a microwave resonator is described in [33]. As this method does not require to paste electrodes, it is especially useful for small crystals and polycrystalline samples. The studied crys- 
tal of RADA $x=0.31$ was placed in the maximum of electric field in cylindrical microwave resonator $T M_{010}$. The c-axis was parallel to electric field lines. The orientation of the sample in the microwave resonator is shown in figure 4 . Thermal contact of the sample with the resonator was provided by special support. The microwave resonator was mounted to the heat exchanging facility in the helium flow cryostat. Assuming that the sample diameter is much smaller than the diameter of the resonator, $\varepsilon^{\prime}$ and $\varepsilon^{\prime \prime}$ were determined from the following expressions:

$$
\begin{aligned}
\varepsilon^{\prime} & =1+0.539 \frac{r_{0}^{2} l}{r^{2} h} \frac{f_{0}-f}{f_{0}} \\
\varepsilon^{\prime \prime} & =0.269 \frac{r_{0}^{2} l}{r^{2} h}\left(\frac{l}{Q}-\frac{l}{Q_{0}}\right),
\end{aligned}
$$

where $r_{0}$ is the radius of the cylindrical resonator; $r$ is the radius of the sample; $l$ and $h$ - the height of the resonator and the sample, respectively; $f_{0^{-}}$and $Q_{0}$ - the frequency and quality factor of the empty resonator; $f$ and $Q$ are the frequency and quality factor of the loaded resonator. Figure 5 presents the results of the temperature dependence of $\varepsilon^{\prime}$ and $\varepsilon^{\prime \prime}$ for RADA, $x=0.31$. There is a clear cusplike maximum on $\varepsilon^{\prime}(T)$ dependence around $65 \mathrm{~K}$ and a maximum on losses $\varepsilon^{\prime \prime}(T)$ dependence at $T_{g}=48 \mathrm{~K}$, typical of proton glass.

In proton glass the electric field $E_{i}$ and polarization $P_{i}$ are strongly related to ordering. Local polarization $P_{i}$ is given by:

$$
P_{i}=\tanh \left[\frac{1}{T}\left(\sum_{j} J_{i, j} P_{j}+E_{i}\right)\right],
$$

a)
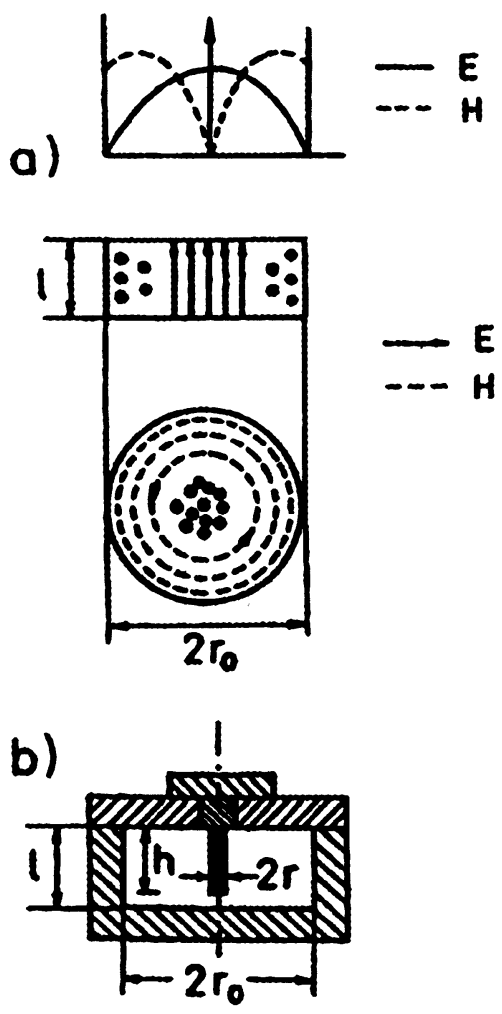

Figure 4. Diagram of the $\mathrm{TM}_{010}$ resonator: a) configuration of the electromagnetic field in the resonator, b) resonator loaded with a sample [33].

where $J_{i j}$ is the interaction energy of pseudospins. There is no long-range order in proton glass, hence the average value of polarization vanishes, i.e. $\langle P\rangle=0$. According to Edwards-Anderson, there is only a short range order within the clusters. The order parameter $q_{E-A}$ can be regarded as square of the average polarization:

$$
q_{E-A}(T)=\frac{1}{N} \sum_{i}\left\langle p_{i}\right\rangle^{2},
$$

where $N$ is the number of dipoles in the cluster and $p_{i}$ is the polarization of a single dipole. In the proton glass state $q_{E-A} \neq 0$. Different models have been developed to describe the proton glass state. The model of clusters by Prelovšek 
and Blinc [51], extended by Matsushita and Matsubara [52], plays an important role in understanding phase diagrams in proton glass systems. The configuration energy is assumed to be given by a
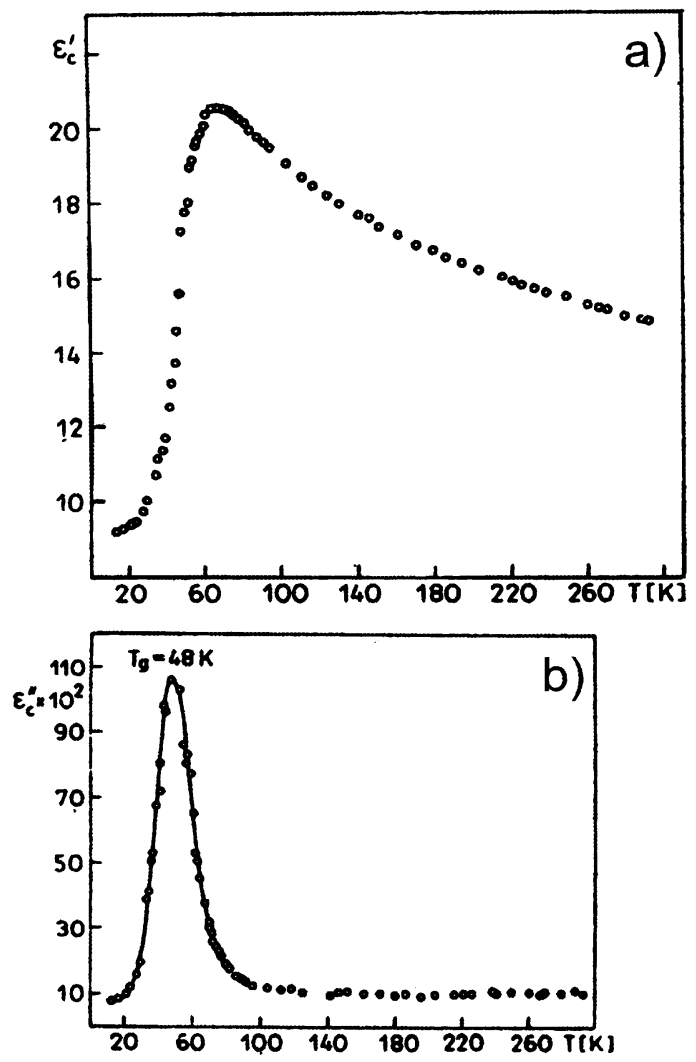

Figure 5. Temperature dependence of the electric permittivity a) $\varepsilon^{\prime}$; b) $\varepsilon^{\prime \prime}$ in RADA $x=0.31$ at $9.2 \mathrm{GHz}$ of the electric measured field $[3,34]$.
Hamiltonian of the form:

$$
H=-\sum_{i>j} J_{i j} \sigma_{i} \sigma_{j}
$$

where pseudospin $\sigma_{i}(i=1,2,3,4)$ is +1 or -1 depending on each of the two possible positions along the hydrogen bond occupied by i-th proton. For a ferroelectric crystal the energy difference between the lateral and up-down ground states equals $\varepsilon_{0}$ and depends on $J$. For an antiferroelectric crystal, besides the energy difference between the two configurations, $-\varepsilon_{o}$, another parameter should be introduced. This extra parameter $w$ accounts for the interaction between two neighbouring parallel hydrogen bonds, which is necessary to establish an antiferroelectric long-range order. For the mixed crystal RADP, considered as a system of clusters ( $j$ is the number of clusters), the Hamiltonian (2) can be written as [52]:

$$
\begin{aligned}
H & =\frac{1}{8} \sum_{j} \varepsilon_{j} \Phi_{j}+\frac{J}{2} \sum_{j} \Psi_{j}, \\
\Phi_{j} & =\left(\sigma_{1}^{j}-\sigma_{3}^{j}\right)+\left(\sigma_{2}^{j}-\sigma_{4}^{j}\right), \\
\Psi_{j} & =\left(\sigma_{1}^{j}+\sigma_{2}^{j}+\sigma_{3}^{j}+\sigma_{4}^{j}\right),
\end{aligned}
$$

$\varepsilon_{j}$ has a different value for each cluster in RADP. A simple Gaussian distribution function for random variable $\varepsilon_{j}$ in the Hamiltonian (3) is assumed:

$$
g(\varepsilon)=\frac{1}{\sqrt{\pi}} \Delta^{-1} \exp \left[-\left(\frac{\varepsilon-\bar{\varepsilon}}{\Delta}\right)^{2}\right],
$$

where $\varepsilon$ is the average of energy $\varepsilon$ depending on the concentration $x$ and $\Delta$ is the energy parameter:

$$
\Delta=\sqrt{2\left\langle(\varepsilon-\bar{\varepsilon})^{2}\right\rangle} .
$$

For the mixed proton-glass crystal RADP $\bar{\varepsilon}$ can take the following values depending on the ammonium concentration $x[52]$ :

$$
\bar{\varepsilon}>0 \quad \text { for } \quad 0<x<0.5
$$




$$
\begin{array}{lll}
\bar{\varepsilon}=0 & \text { for } & x=0.5, \\
\bar{\varepsilon}<0 & \text { for } & 0.5<x<1 .
\end{array}
$$

The glass transition temperature $T_{g}$ is given by [52]:

$$
\left(\frac{k_{\mathrm{B}} T_{g}}{\Delta}\right)=\frac{1}{8} \frac{1+2 y^{2}}{(1+2 y)^{2}}
$$

where:

$$
y=\exp \left(-\frac{-\bar{\varepsilon}}{k_{\mathrm{B}} T}\right) .
$$

The average of $\bar{\varepsilon}(x)$ is given by [52]:

$$
\bar{\varepsilon}(x)=\varepsilon P_{+}(x)+0 \cdot P_{o}(x)-\varepsilon P_{-}(x),
$$

where $P_{+}(x), P_{o}(x)$ and $P_{-}(x)$ are three $x$-dependent probability functions for finding a cluster in three groups: ferro, neutral and antiferro group, respectively. This function is plotted in figure $6 \mathrm{a}$. The broken lines are $\varepsilon(x)$ dependences for different RADA crystals.
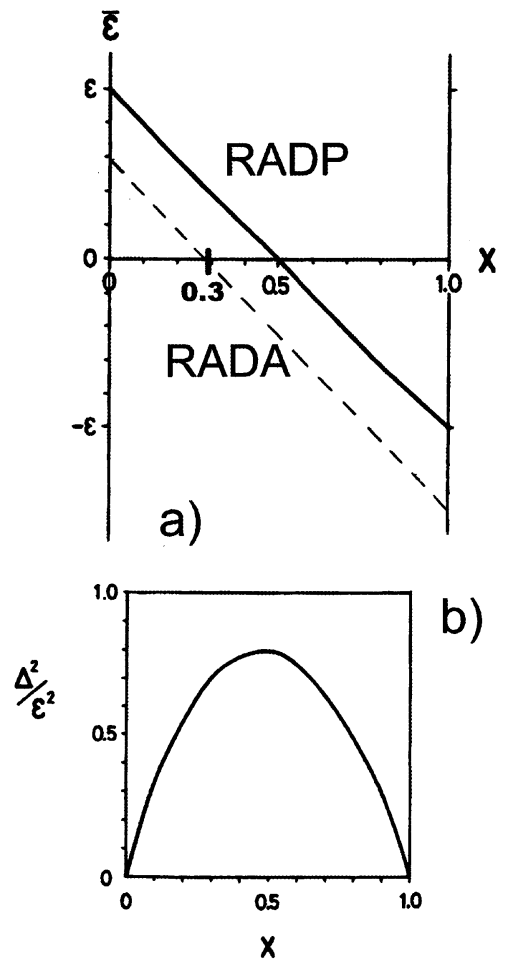

Figure 6. Concentration dependence of: a) the average energy between up-down and lateral; b) $\left(\Delta / \varepsilon_{o}\right)^{2}$ (see equation 4) [52].

The root mean square as a function of $x$ (figure $6 \mathrm{~b}$ ) is defined as [52]:

$$
\frac{\Delta^{2}(x)}{\varepsilon^{2}}=2\left\{1-P_{o}(x)-\left[P_{+}(x)-P_{-}(x)\right]^{2}\right\} .
$$

The paraelectric-ordered phase transitions of the components of mixed RADP crystals do not differ much. RDP has the Curie temperature $T_{c}=148 \mathrm{~K}$ [30], while for antiferroelectric ADP the Néel temperature equals $T_{N}=150 \mathrm{~K}$ [31]. Thus, for $x=0.5$ composition of RADP the average energy is zero, $\varepsilon=0$. In the RADA crystal the temperatures of paraelectric-ordered phase transitions are very different for the both components $\left(\mathrm{RDA}, T_{c}=110 \mathrm{~K}[32]\right.$, and $\mathrm{ADA} T_{N}=216 \mathrm{~K}$ [31]). This difference implies that the RADA phase diagram should be asymmetric because $\varepsilon=0$ falls on approximately $x=0.3$ concentration. Microwave dielectric measurements for different NH4 concentrations $x$, shown in figure 7 , support this conclusion. The phase diagram of RADA, thus determined, (figure 8), differs from that of RADP. The phase diagram of RADA is asymmetric with the glass state for NH4 concentration of $0.1<$ $x<0.5$. The temperature of the transition to the proton glass state increases with frequency of the measured electric field, but is not $\mathrm{NH}_{4}$ concentration dependent, like for RADP. This asymmetry of the RADA phase diagram from microwave studies was confirmed by Kim and 
Kwun [7] in dielectric measurements at $700 \mathrm{~Hz}$ to $1 \mathrm{MHz}$ and the existence of the proton glass state was limited to the concentration of $0.13<x<0.49$ (figure 9).
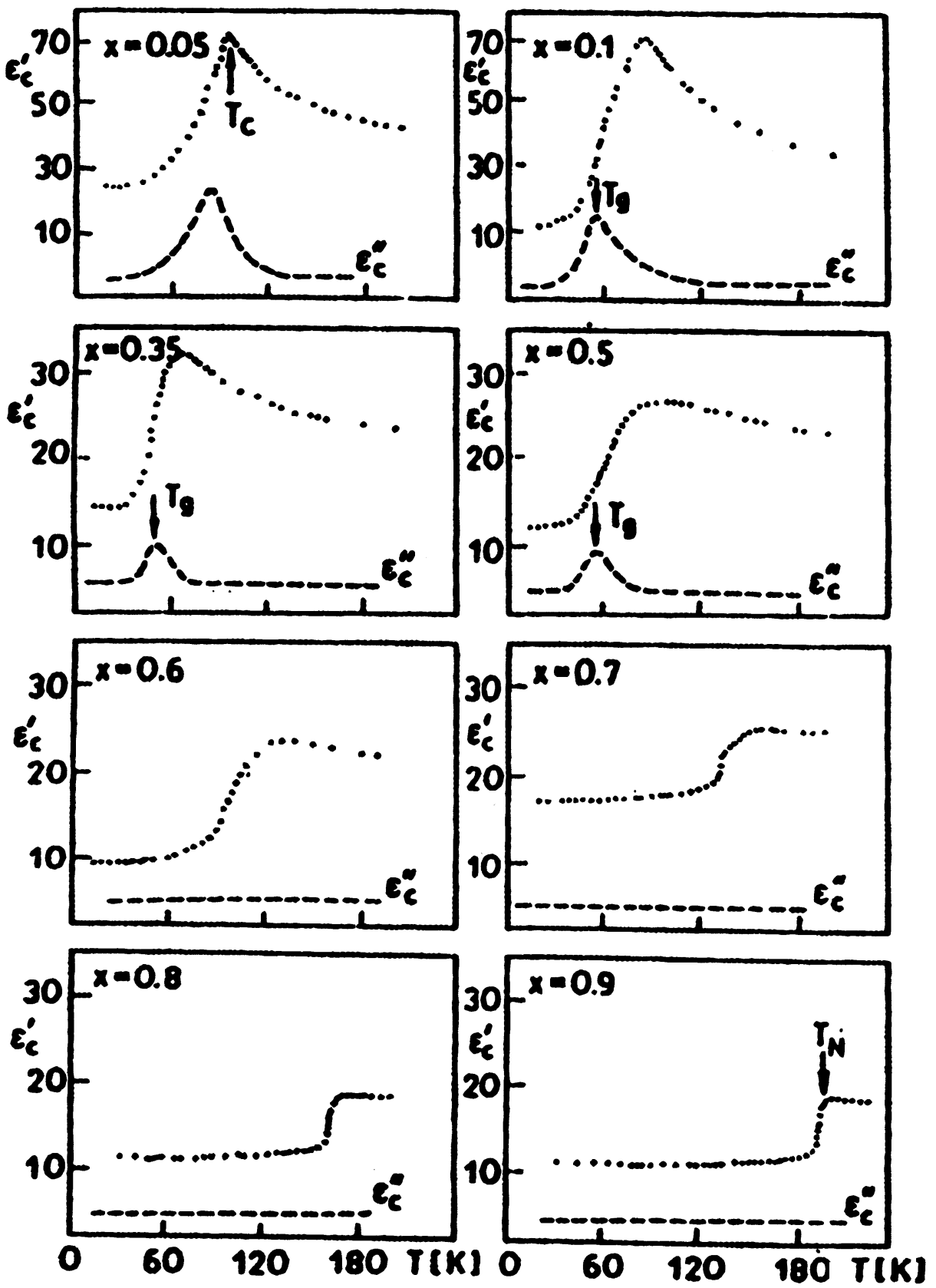

Figure 7. The temperature and concentration $\mathrm{x}$ dependence of the electric permittivity $\varepsilon_{c}^{\prime}$ and $\varepsilon_{c}^{\prime \prime}$ in RADA $x=0.31$ at $9.2 \mathrm{GHz}$ of the measured electric field [4].

Further studies of the proton glass state in RADA revealed dispersion of electric permittivity in the transition from the paraelectric to the proton glass phase. The dielectric properties of the RADA crystal $(x=0.35)$ were investigated for two perature to $T_{f}>70 \mathrm{~K}$. Deviation of $\varepsilon^{\prime}(T)$ from the Curie-Weiss law determines 


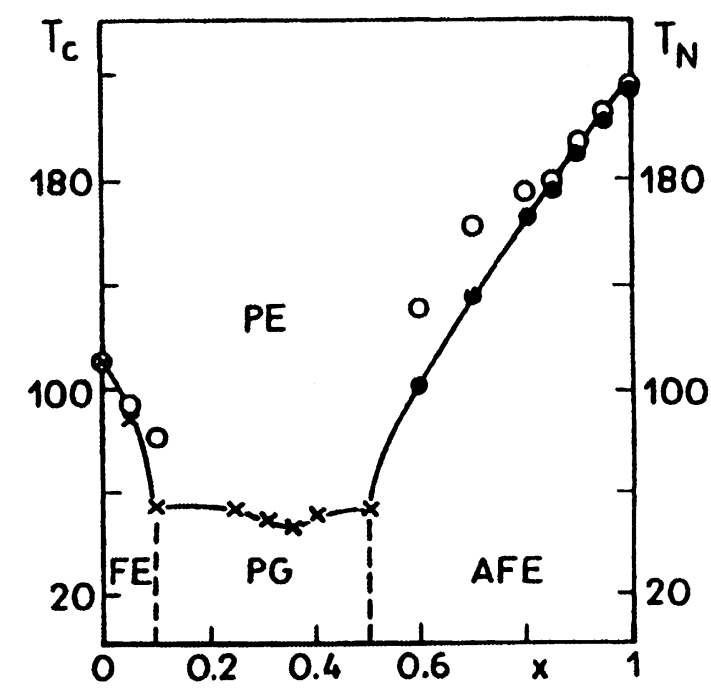

Figure 8. The phase diagram of RADA. $\mathrm{PE}, \mathrm{FE}, \mathrm{AFE}$, and $\mathrm{PG}$ denote paraelectric, ferroelectric, antiferroelectric and proton glass phases, respectively. The open circles and crosses denote maximum of $\varepsilon_{c}^{\prime}$ and $\varepsilon_{c}^{\prime \prime}$, respectively. The full circles denote the middle of the phase transition region from the $\varepsilon_{c}^{\prime}(T)$ dependence [4].

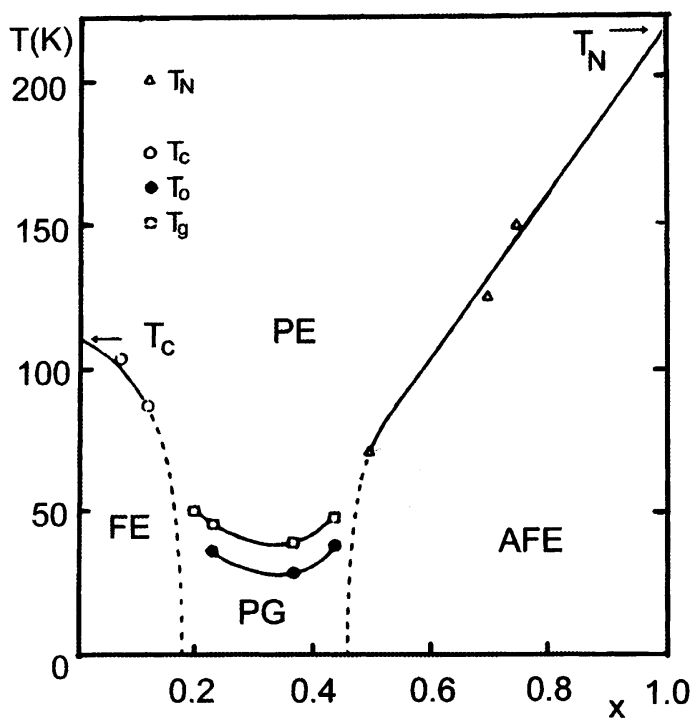

Figure 9. The phase diagram of RADA done by Kim and Kwun [7] from electric permittivity $\varepsilon_{a}^{\prime}$ and $\varepsilon_{a}^{\prime \prime}$.
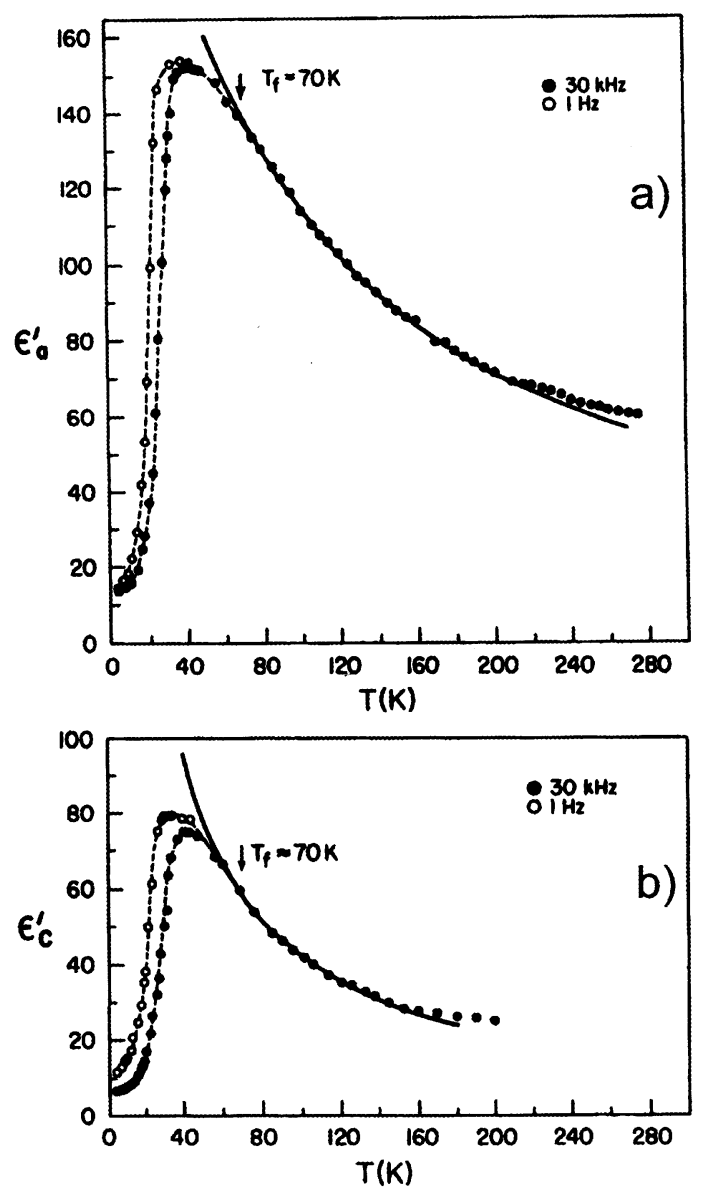

Figure 10. Temperature dependence of the dielectric permittivity in RADA $x=$ 0.35: a) $\varepsilon_{a}^{\prime}(T)$; b) $\varepsilon_{c}^{\prime}(T)$. Solid lines present fits to the Curie-Weiss law [6].

samples [6]: along a and $\mathrm{c}$ axis of the crystal. Figure 10 shows a real part of electric permittivity data for both crystal orientations. For the both directions a typical proton glass behaviour is observed. As the temperature lowers from the room temperature to about $40 \mathrm{~K}, \varepsilon^{\prime}$ initially increases. After a cusp-like maximum $\varepsilon^{\prime}$ decreases to the value of approximately 10 at temperature $T=3 \mathrm{~K}$. Electric permittivity $\varepsilon^{\prime}$ can be well described by the Curie-Weiss law in the paraelectric phase from the room tem- 
temperature $T_{f}$. At this temperature a short-range order is established in certain regions of the crystal - clusters start to be formed. On decreasing the temperature, the volume of each cluster increases to Vogel-Fulcher temperature $T_{o}$ (equation 5), according to the following equation first applied to proton glass by Courtens [36]:

$$
\nu_{c}=\nu_{o} \exp \left(\frac{-E_{c}}{T-T_{o}}\right)
$$

where $T_{o}$ is Vogel-Fulcher freezing temperature, $E_{c}$ is a cut-off energy in the temperature unit, $\nu_{o}$ is an attempt frequency. The temperatures $T_{g}$ of the freezing electric dipoles reorientation within the cluster have been determined for each measuring frequency from the maximum of $\varepsilon^{\prime \prime}(T)$. At $T_{o}$, the reorientation of electric dipoles within the cluster becomes frozen. Dielectric dispersions of $\varepsilon^{\prime}(T)$ and $\varepsilon^{\prime \prime}(T)$ have been demonstrated for $T<40 \mathrm{~K}$ (figure 10). As the frequency of the measured electric field increases, the maximum of $\varepsilon^{\prime \prime}(T)$ shifts to a higher temperature.

The proton glass state was also observed in $\mathrm{K}_{1-x}\left(\mathrm{NH}_{4}\right)_{x} \mathrm{H}_{2} \mathrm{AsO}_{4} x=0.40$, (KADA $x=0.40$ ) [35]. The substitution of Rb by K does not influence the properties of the proton glass state significantly. The temperature dependence of $\varepsilon_{a}^{\prime}$ for KADA $x=0.40$ is very similar to that of RADA.

\section{Coexistence of paraelectric/proton-glass and ordered long- range phases}

\subsection{Undeuterated glass RADA}

Detailed dielectric studies of mixed RADA crystals for different $x$ concentrations have shown that for very small, as well as for very large $x$ concentrations the crystals are exclusively in a ferroelectric /or antiferroelectric phase. The first evidence of the coexistence of paraelectric, glass and ordered ferroelectric phases was given by Trybuła, Schmidt and Drumheller [8] for RADA $x=0.12 ; 0.15$ and 0.20. This result was confirmed by Eom et al. [9] in dielectric and laser optical studies. The latter shows that the orthorhombic symmetry - optically biaxial characteristic of a ferroelectric phase is preserved up to the lowest temperature at which the glass state is present. The crystal in the glass state is tetragonal (optically uniaxial), like in a paraelectric phase. It seems that the glass state exists independently of the ferroelectric one.

Temperature dependences of the real part of electric permittivity $\varepsilon_{a}^{\prime}$ in RADA for different ammonium concentrations $x$, revealing the coexistence of glass and ferroelectric phases, are shown in figure 11 and 12 [8]. The ammonium concentration was determined by measuring the rubidium content with flame atomic-absorption spectroscopy. In contrary to RADP, in RADA the concentration of rubidium $(1-x)$ in solution equals the concentration in the crystal. The following concentrations were studied: $x=0, x=0.12 \pm 0.01, x=0.15 \pm 0.01$ and $x=0.20 \pm 0.01$. For $x=0.12$ and $x=0.15$ there are two transitions: at $T_{c}$ to a ferroelectric phase 


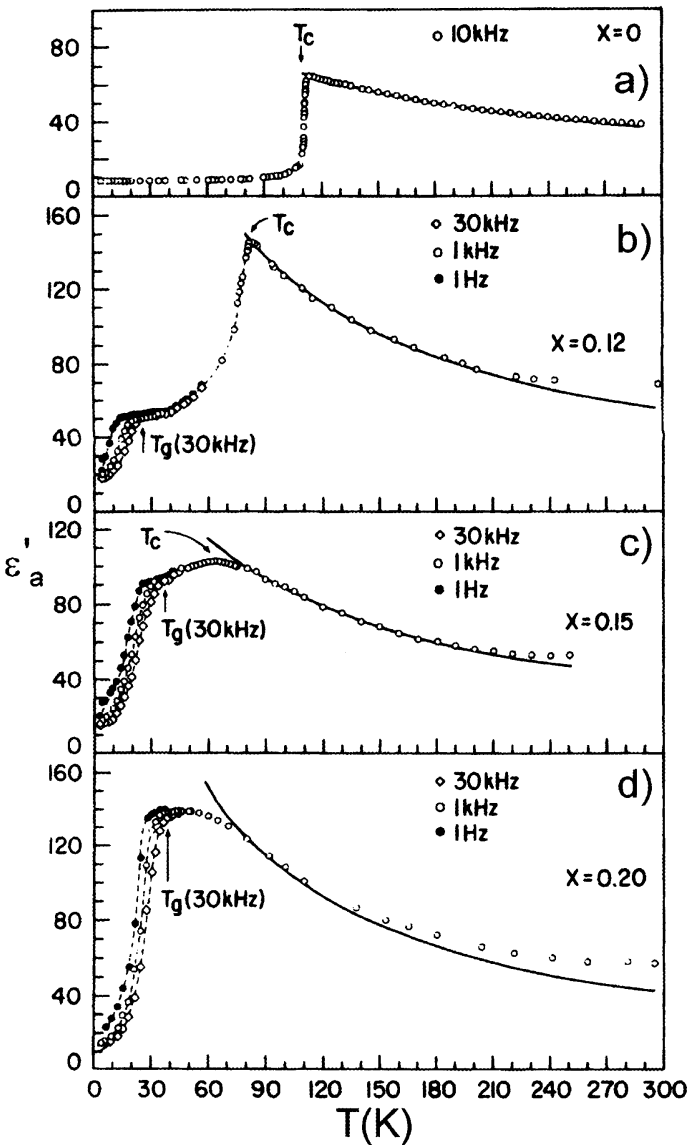

Figure 11. Temperature dependence of the real part of the electric permittivity $\varepsilon_{a}^{\prime}$ in a) RDA; b) RADA $x=0.12$; c) RADA $x=0.15 ;$ d) RADA $x=0.20$ [8].

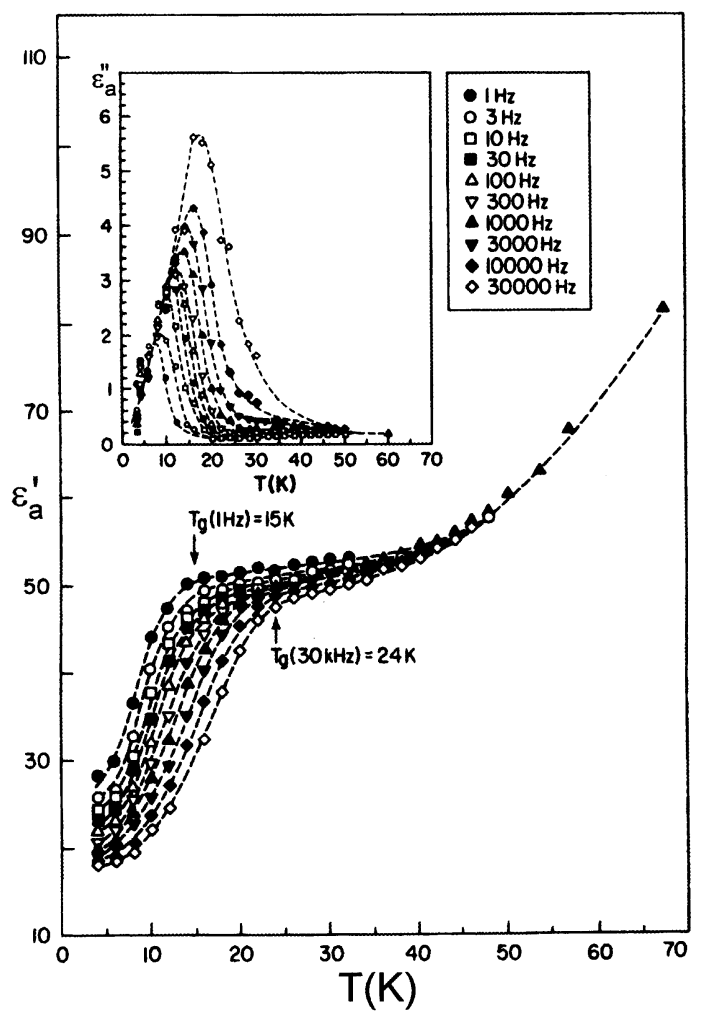

Figure 12. Dielectric dispersion of $\varepsilon_{a}^{\prime}$ in RADA $x=0.12$ in the proton glass regime [8].

and at $T_{g}$ to a glass phase. The transition to the ferroelectric phase is not frequency dependent, while that to the glass state displays dispersion both in $\varepsilon^{\prime}$ and $\varepsilon^{\prime \prime}$ (figure 12). The measurements along the a axis of the crystal, perpendicular to ferroelectric axis c, excluded the possibility of dispersion, typical of KDP ferroelectrics related to the freezing of the dynamics of the domains walls. Such dispersion does not exist along the $a$ axis. The RADA results in figure 11 clearly show two phase transitions. In the case of the coexistence of ferroelectric and glass phases, Tg for the given frequency of the measured electric field is lower than $\mathrm{Tg}$ at the same frequency for RADA exhibiting only the glass state. This observation suggests that the volume of the cluster with a short-range order in crystals with phase coexistence is smaller than the volume of the clusters in crystals in the glass state only. Moreover, because of the shorter correlation length, the dynamics of the clusters is less hindered. Howell, Pinto and Schmidt [10] have shown that the coexistence of ferroelectric / glass phases exists even for lower concentrations, i.e. $\mathrm{x}=0.05$. Recent measurements up to $0.4 \mathrm{~K}$ by Trybuła et al. [37] reveal 


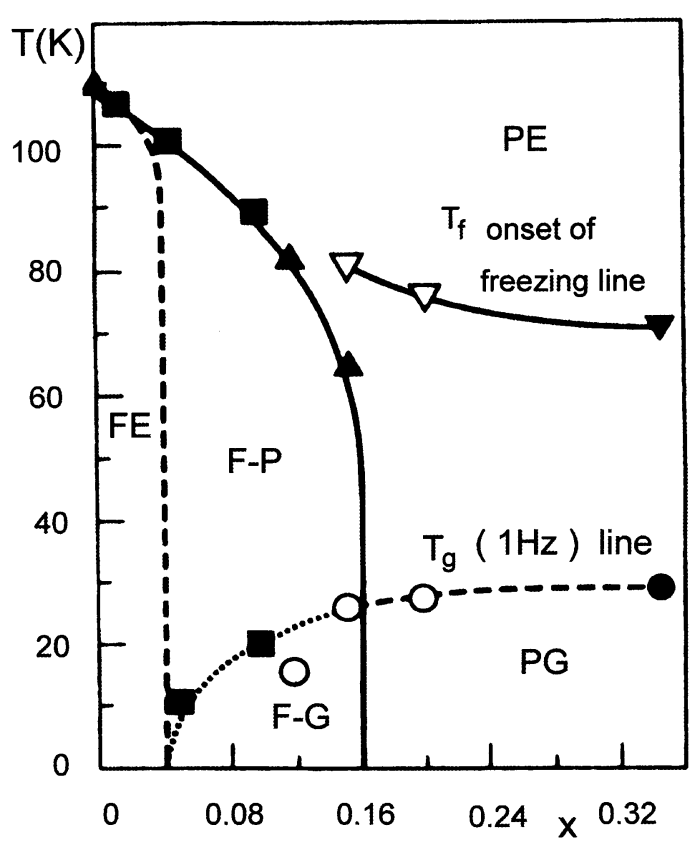

Figure 13. Part of the phase diagram of RADA: PE- paraelectric phase; FEferroelectric phase; $\mathrm{PG}-$ proton glass regime; F-P-coexistence of paraelectric and ferroelectric phases; , F-G »coexistence of ferroelectric and proton-glass phases $[8,10]$.

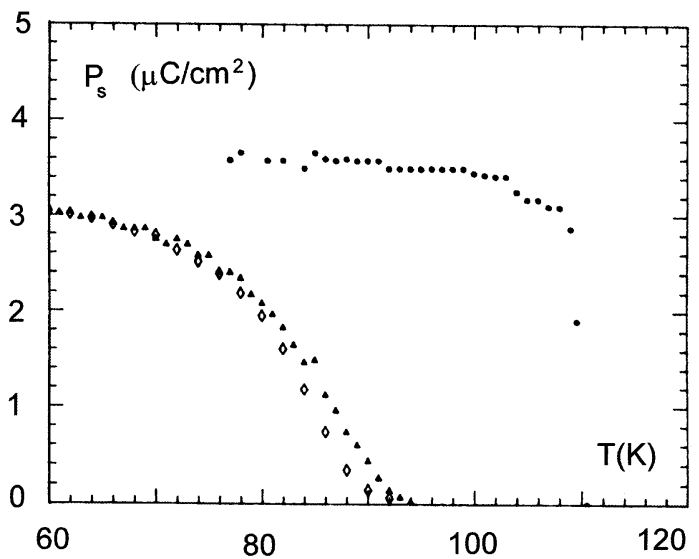

Figure 14. Spontaneous polarization obtained from saturated hysteresis loops in $\mathrm{RDA} \mathrm{x}=0(\bullet)$ and RADA $x=0.08(\mathbf{\Delta})$ as a function of temperature. The open diamond $(\diamond)$ symbol represents spontaneous polarization obtained from equation (6) and figure 15 for RADA $x=0.08$ [12]. that such coexistence can be observed even for $x=0.01$. The part of the RADA phase diagram presenting the coexistence of ferroelectric/glass phases is shown in figure 13 .

The coexistence of ferroelectric and proton glass states was also observed in other isomorphic ferroelectric-antiferroelectric mixed crystals: $\mathrm{K}_{1-x}\left(\mathrm{NH}_{4}\right)_{x} \mathrm{H}_{2} \mathrm{AsO}_{4} \quad x=0.23$ [38], $\mathrm{K}_{1-x}\left(\mathrm{NH}_{4}\right)_{x} \mathrm{H}_{2} \mathrm{PO}_{4}$ [39], and also for deuterated DRADA $[11,13]$.

The coexistence of paraelectric/proton glass and ferroelectric orders below the glass transition temperature $T_{g}$ was confirmed by spontaneous polarization measurements in deuterated DRADA an undeuterated RADA crystals by Pinto et al. [12]. The temperature dependence of $P_{s}$ for a ferroelectric crystal RDA and mixed RADA $x=0.08$ was determined from a saturated hysteresis loop in the standard Sawer-Tower circuit. Figure 14 presents the results for a nondeuterated crystal. A RDA crystal exhibits a distinct jump of spontaneous polarization at $T_{c}=110 \mathrm{~K}$, typical of the first order phase transition. The spontaneous polarization reaches the value of $3.6 \pm 0.5 \mu \mathrm{C} / \mathrm{cm}^{-2}$ at the temperature far below $T_{c}$. For a mixed crystal RADA $x=0.8$, below $T=94 \mathrm{~K}$, there is a gradual increase of $P_{s}$. The maximum value of $P_{s}$ attained in a mixed c rystal is lower than that of a RDA crystal. Spontaneous polarization of a mixed crystal can be thus described by the following expression [12]:

$$
P_{\text {sd }}(T)=P_{\text {so }}\left[\frac{\varepsilon_{1}^{\prime}(T)}{\varepsilon_{1}^{\prime}(T)+\varepsilon_{2}^{\prime}(T)}\right],
$$

where $P_{\text {sd }}$ is the spontaneous polarization of the mixed crystal, $P_{\text {so }}$ is the 
maximum spontaneous polarization of the pure crystal RDA well below $T_{c}$; $\varepsilon_{1}^{\prime}$ and $\varepsilon_{2}^{\prime}$ electric permittivity values marked in figure 15 which gives temperature dependences of $\varepsilon^{\prime}$ for proton-glass RADA $x=0.40$ and RADA $x=0.08$. Spontaneous polarization of the mixed crystal is equal to the spontaneous polarization in the pure RDA crystal well below $T_{c}$ multiplied by the fraction of the mixed crystal that becomes ferroelectric below $T_{c}$. Similar results were obtained by Pinto et al. [12] for the deuterated D-RADA $x=0.08$ crystal. The maximum value of the spontaneous polarization in mixed RADA

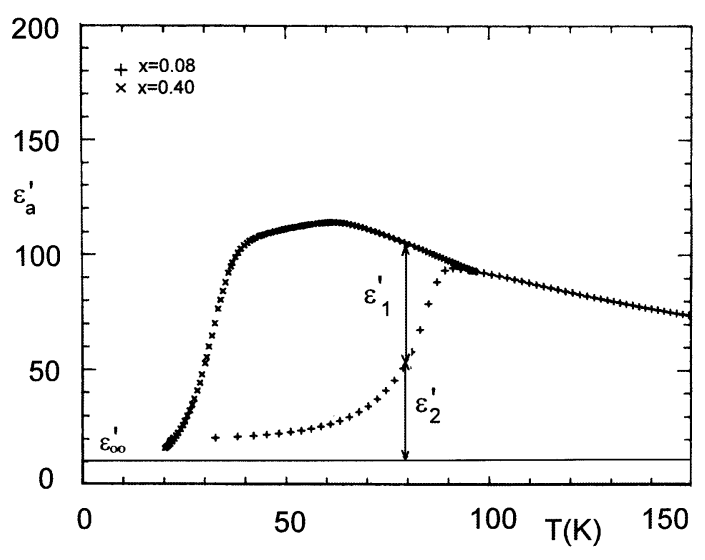

Figure 15. Temperature dependence of the electric permittivity e»a for RADA $x=0.08$ and $x=0.40$ at $1 \mathrm{kHz}\left(\varepsilon_{\infty}^{\prime}\right.$ is assumed to be 10) [12].

$x=0.8$, and its deuterated counterpart is lower than that of the pure crystal. This indicates that at lower temperatures there are still paraelectric clusters closely interlocked with ferroelectric clusters.

\subsection{Deuterated glass DRADA}

Deuteration shifts the transition temperature $T_{c}$ upward from $110 \mathrm{~K}$ for RDA to $170 \mathrm{~K}$ for DRDA or, in antiferroelectrics, the Néel temperature $T_{N}$ from 216 for ADA to $304 \mathrm{~K}$ for DADA and increases the value of the spontaneous polarization $P_{s}$ compared with the undeuterated crystal [40]. The studies of deuteron glass have revealed that, like in a undeuterated crystal, the state of glass may coexist with the ferroelectric or antiferroelectric order [10-14]. Deuteration leads to the narrowing of glass existence range to $0.2 \leqslant x \leqslant 0.35$ [14-15,41].

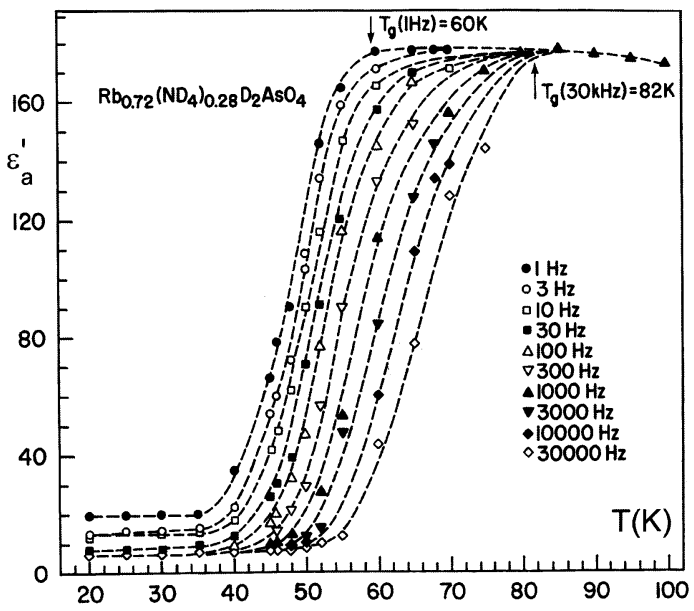

Figure 16. Dispersion of electric permittivity $\varepsilon_{a}^{\prime}(T, \nu)$ for DRADA $x=0.28$.

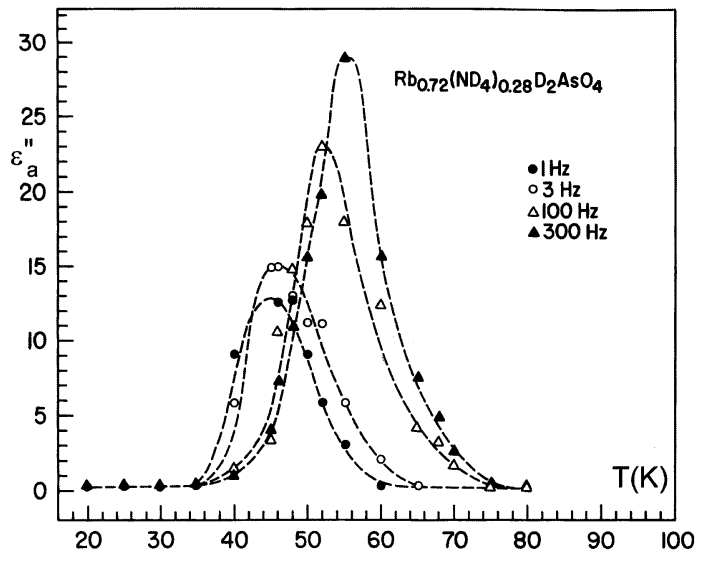

Figure 17. Dispersion of electric permittivity $\varepsilon_{a}^{\prime \prime}(T, \nu)$ for DRADA $x=0.28$. 
The complex dielectric permittivity $\varepsilon_{a}^{\prime}(T)$ and $\varepsilon_{a}^{\prime \prime}(T)$ of DRDA for $x=0.28$ in the deuteron glass range, typical of the glass state, are presented in figures 16 and 17. The dielectric permittivity $\varepsilon_{a}^{\prime}(T)$ of deuterated DRADA for $x=0.39$ studied by Trybuła et al. [11] is shown in figure 18. A typical behaviour attributed to the transition from the paraelectric to antiferroelectric state is marked at $T_{N}=127 \mathrm{~K}$. This phase transition takes place at the same temperature for different frequencies of the measured electric field. Precise measurements for the temperature below 100 K show the occurrence of dispersion of the permittivity $\varepsilon_{a}^{\prime}(T)$ in the temperature range from $20 \mathrm{~K}$ to $90 \mathrm{~K}$ (figure 19). Analysis of the shape of the temperature

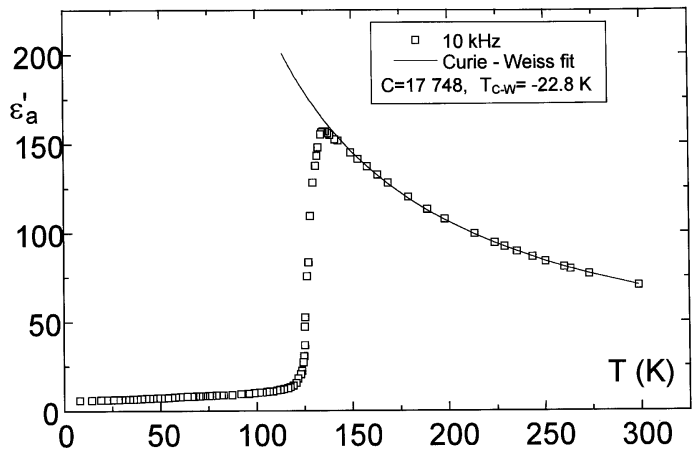

Figure 18. Temperature dependence of the real part of the electric permittivity e»a for DRADA $x=0.39$, at the frequency of the measuring electric field $\nu=10 \mathrm{kHz}[11]$.

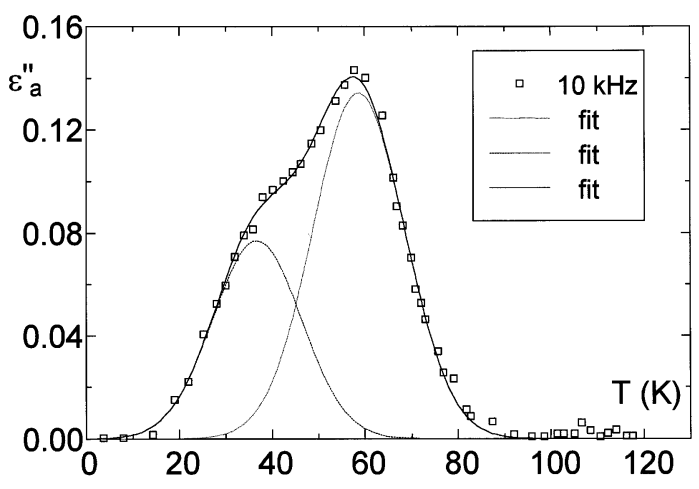

Figure 20. The fit of the experimentally obtained $\varepsilon_{a}^{\prime \prime}(T, \nu)$ data for DRADA $x=0.39$ using two Gaussian-shape lines (at $\nu=10 \mathrm{kHz}$ ). The contributions of two different relaxation mechanisms are marked [11].

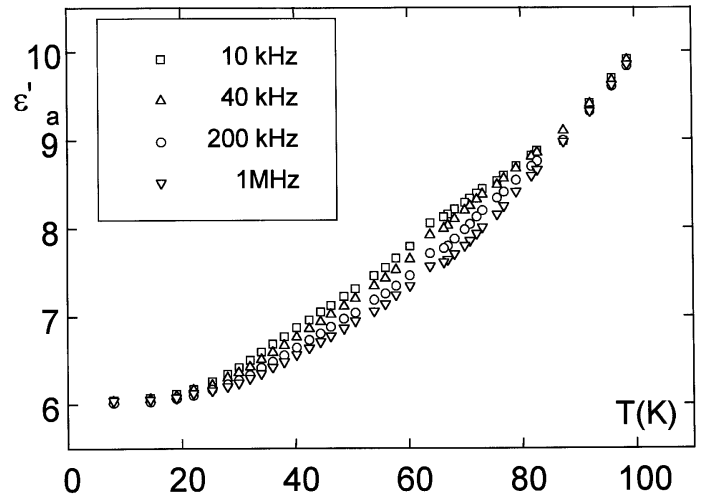

Figure 19. Dispersion of electric permittivity $\varepsilon_{a}^{\prime}(T, \nu)$ for DRADA $x=0.39$ [11].

dependence of $\varepsilon_{a}^{\prime \prime}(T)$ (figure 20) proves the existence of phases of a short-range order. In the antiferroelectric phase, the values of $\varepsilon^{\prime \prime}$ do not change with temperature, as a result of the opposite dipolar moments of the two sublattices of the crystal due to the ordering of deuterons in the hydrogen bond O-D ... and the formation of the lateral Slater configuration. The temperature and frequency dependence of $\varepsilon^{\prime \prime}$, two orders of magnitude smaller than those characteristic of the concentration range in which deuteron glass can exist, provides information that the regions of the glass state are formed where a long-range antiferroelectric order disappears. A complex temperature 
dependence of $\varepsilon_{a}^{\prime}(T, \nu)$ indicates two kinds of electric dipolar relaxation. One of these mechanisms can be described by the Vogel-Fulcher temperature (equation 5) with the parameters: $T_{0}=25.7 \mathrm{~K}, E_{c}=105.6 \mathrm{~K}$ and $\nu_{o}=$ $1.16 \cdot 10^{8} \mathrm{~Hz}$, and is typical of clusters with a short-range order characteristic of proton (deuteron) glass. The second relaxation mechanism is the thermally activated Arrhenius dipolar reorientation with activation energy $E_{c}=1105$ $\mathrm{K}$ and frequency $\nu_{o}=1.44 \cdot 10^{12} \mathrm{~Hz}$. The Arrhenius-type relaxation is related to free dipoles which are released from the melting long-range ordering but have not managed to form a cluster yet. Similar behaviour of relaxation, described by the Arrhenius equation, was reported by Hutton et al. [42] for pro-

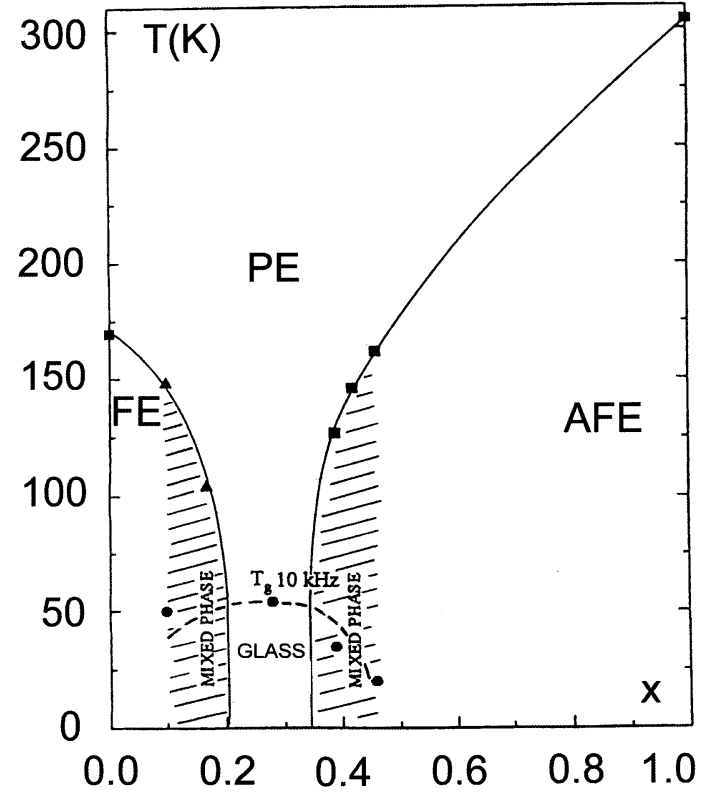
ton glass which is a mixture of antiferroelectric betaine phosphate $(\mathrm{BP}):(\mathrm{CH})_{3} \mathrm{NCH}_{2} \mathrm{COO} \cdot \mathrm{H}_{3} \mathrm{PO}_{4}$, and ferroelectric betaine phosphite (BPI): $(\mathrm{CH})_{3} \mathrm{NCH}_{2} \mathrm{COO} \cdot \mathrm{H}_{3} \mathrm{PO}_{3}$ and in which the hydrogen bonds between $\mathrm{PO}_{3}$ or $\mathrm{PO}_{4}$ groups form quasi-one dimensional chains. The Arrheniustype relaxation is typical of strong glass [43] characterized by a low density of configurational states in their potential energy.

Figure 21 presents a new phase diagram of DRADA displaying the coexistence of paraelectric/proton glass and ferroelectric (antiferroelectric) phases.

\section{Pressure dependence of a proton glass phase}

The first high-pressure studies in RADP mixed crystals were carried out by Samara et al. [45-46]. The effect of pressure was expected to influence the glassy state via the pressure dependence on the hydrogen-bond length. These studies have led to a much better understanding of the nature of the competing inter-molecular and intra-molecular interactions which are responsible for the establishment of a long-range order. Pressure modifies interactions responsible for a short-range correlation; therefore, the results of pressure dependence provide new insights into the formation and properties of the glassy state. In the family of KDP crystals the proton moves in a double-well potential along the hydrogen bond. In the high- temperature tetragonal paraelectric phase the protons are disordered in the potential wells leading to an effectively symmetric hydrogen bond. In the glass state the proton freezes in one or another potential minimum; this results in an elongated asymmetric hydrogen bond. Pressure reduces the $\mathrm{H}$-bond length and 
favours a more symmetric bond with a lower energy barrier, which in turn leads to a lower glass transition temperature. For a sufficiently high pressure, the $\mathrm{H}-$ bond will become effectively symmetric, so that there will be no order and the glassy state will vanish. The results of Samara for the RADP with $x=0.48,72 \%$ deuterated crystal [45] and RADP with $x=0.50$ [46] show the lowering of the glass temperatures $T_{g}$, as presented in figure 22 . The results for c and a crystallographic

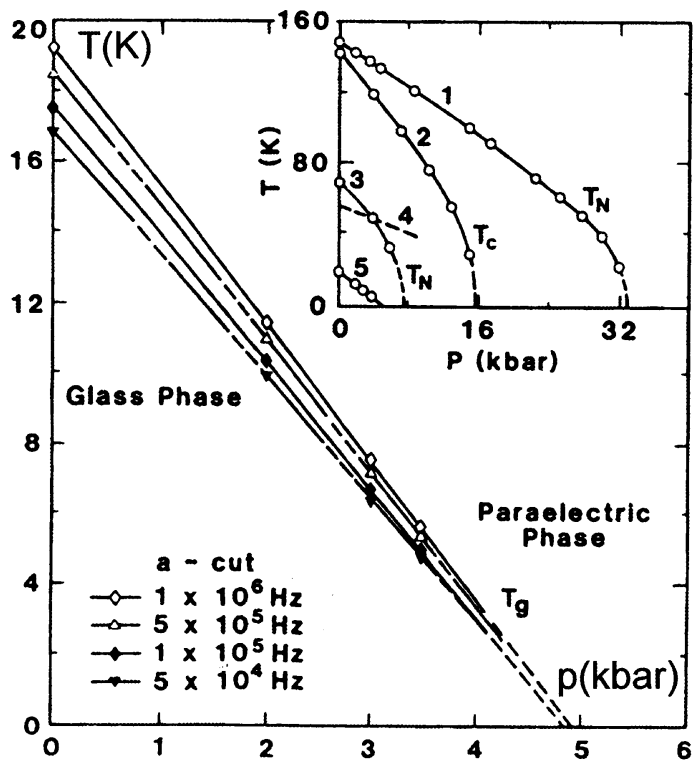

Figure 22. Temperature-pressure phase diagram for an RADP crystal. Insert: comparison of the pressure dependences of the dynamic glass $T_{g}$, ferroelectric $T_{c}$, and antiferroelectric TN transition temperatures for several crystals of RADP. Curves $1-5$ correspond to $x=1 ; 0 ; 0.8$; $0.48(72 \% \mathrm{D})$; and 0.5 , respectively [46]. axes were qualitatively similar. Comparison of a pressure-induced suppression of the glassy state in a RADP mixed crystal with the suppression of the ferroelectric state in RDP and the antiferroelectric state in ADP shows an important difference between the glassy transition and ferroelectric or antiferroelectric transitions. For pure ferroelectric or antiferroelectric crystals the magnitude of slope $\mathrm{d} T_{c, N} / \mathrm{d} p$ increases with pressure at high pressure values. The data strongly suggest that the transition vanishes at an infinite slope, i.e. $\mathrm{d} T_{c, N} / \mathrm{d} p \rightarrow-\infty$ as $T_{c, N} \rightarrow 0$. The results of glassy RADP $x=0.5$ are different. Glass transition temperature $T_{g}$ decreases linearly with pressure up to temperature $5 \mathrm{~K}$ with no hint of any impending increase in the magnitude of $\mathrm{d} T_{g} / \mathrm{d} p$ at a lower temperature. There are no data below $4 \mathrm{~K}$, and only linear $T_{g}(p)$ extrapolation to higher pressures is given. Samara supposes that the proton glass phase will disappear at 5 kbar. Up to this time there is no experimental evidence of a linear or nonlinear $T_{g}(p)$ response below $4 \mathrm{~K}$. Samara believes that this linear dependence is most likely the evidence of the nonequilibrium nature of the glass transition in RADP glass crystals. There is a large hydrogen-isotope effect not only on $T_{g}$ but also on its pressure derivative. The magnitude of $\mathrm{d} T_{g} / \mathrm{d} p$ decreases from -3.6 to $-2.0 \mathrm{~K} / \mathrm{kbar}$ on deuteration. The higher $T_{g}$ and smaller $\mathrm{d} T_{g} / \mathrm{d} p$ for deuterated glass are due to the fact that a deuteron is located deeper in the potential well than a proton, and there is a much lower probability for tunneling between two potential wells along the O-D ...O bond.

The effects of hydrostatic pressure on the dielectric properties and phase transitions were investigated in a RADA crystal by Samara and Schmidt [16] for the compositions in the coexistence region of proton-glass and ferroelectric or antifer- 
roelectric orders. Figure 23 shows that this crystal is inhomogeneous, containing a sufficiently large region (about $4 \%$ of the sample's volume) of pure RDA, as indicated by $T_{c 1}=110$ K. The second transition at $T_{c 2}=$ $90 \mathrm{~K}$ is related to the presence of RADA for $x=0.1$. Glass transition temperature $T_{g}$ is marked at $30 \mathrm{~K}$. The pressure derivatives of $T_{c}$ for the paraelectric-ferroelectric transitions in RDA (-4.6 K/kbar) are about twice as large as those of $T_{N}$ for the paraelectricantiferroelectric ones in ADA (-1.97 $\mathrm{K} / \mathrm{kbar}$ ). The pressure derivative of $T_{g}$ for the paraelectric-proton glass transition $(-2.2 \mathrm{~K} / \mathrm{kbar})$ is of about the same

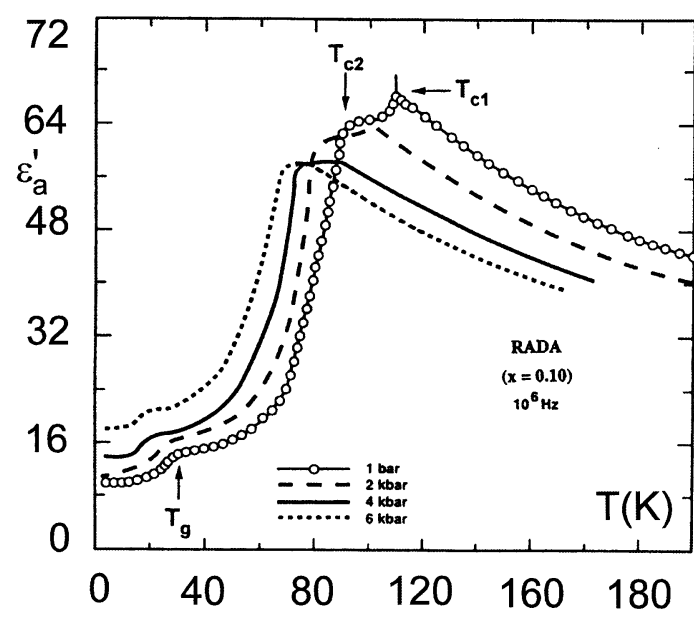
magnitude as for ADA suggesting that

Figure 23. Temperature dependence of the $\varepsilon_{a}^{\prime}$ electric permittivity in RADA $x=$ 0.1 at different applied pressure [16].

the compressibility of ADA clusters in RADA determines the glass transition. The $T_{g}$ is weakly dependent on the composition over most of the region of a proton glass phase. Samara's results indicate that pressure derivative $\mathrm{d} T_{g} / \mathrm{d} p$ is also essentially independent of the composition. The decrease of $T_{c}, T_{N}$, and $T_{g}$ with pressure results from an increase in the tunnelling frequency and a decrease in the dipolar interaction which is long-range in the case of ferroelectric and antiferroelectric phases and short-range (probably antiferroelectric) in proton glass.

\section{External dc electric field dependence of electric permittivity in RADA}

The temperature dependence of the field-cooled (FC), zero field-cooled (ZFC) and field-heated $(\mathrm{FH})$ static permittivity $\varepsilon^{\prime}$ was studied in deuterated DRADP crystals by Levstik et al. [47] and in Deuterated Rubidium Ammonium Dihydrogen Arsenate DRADA $x=0.28$ by Pinto et al. [44]. Unlike magnetic spin glass, where only random-bond type interactions exist [48], proton and deuteron glass are characterized by the presence of random bonds and a random field [49-50]. The random bias electric field is due to the random sites of the $\mathrm{NH}_{4}$ (or $\mathrm{ND}_{4}$ ) groups; this leads to a random freeze-out of the acid proton (or deuteron) in the hydrogen bonds $\mathrm{O}-\mathrm{H} \cdots \mathrm{O}$ as temperature is lowered below $T_{g}$. Because of this field the Edwards-Anderson order parameter $q_{E A}$ (see equation 1) is nonzero in the whole temperature range. In magnetic spin glass systems the $q_{E A}$ is zero above glass transition temperature and nonzero below $T_{g}$.

The static dielectric response of deuteron glass to the external dc electric field depends on the history of the sample. It is important how the low temperature glass phase (nonergotic) is reached. Above the glass phase the field-cooled $\varepsilon_{F C}^{\prime}$ 
and zero field-cooled $\varepsilon_{Z F C}^{\prime}$ electric permittivities are the same, whereas below $T_{g}$, in general $\varepsilon_{F C}^{\prime}>\varepsilon_{Z F C}^{\prime}$. The DC electric field increases the value of a static permittivity. The field-cooled electric permittivity $\varepsilon_{F C}^{\prime}$ retains the same value as temperature is lowered below $T_{g}$ and is constant at the temperature decrease due to the gradual freeze-out of the acid proton or deuteron in the hydrogen $\mathrm{O}-\mathrm{H} \cdots \mathrm{O}$ bond. On switching the external dc field off, remanent polarization is observed which vanishes as temperature is raised above $T_{g}$.

\section{Conclusions}

Dielectric studies of the proton state have contributed to a better understanding of the phenomena in mixed crystals $\mathrm{Rb}_{1-x}\left(\mathrm{NH}_{4}\right)_{x} \mathrm{H}_{2} \mathrm{AsO}_{4}$ and deuterated $\mathrm{Rb}_{1-x}\left(\mathrm{ND}_{4}\right)_{x} \mathrm{D}_{2} \mathrm{AsO}_{4}$. After first observation of the glass state in dielectric data for RADA $x=0.31$ in 1986 [3], further measurements in the microwave region by Trybuła, Stankowski and Blinc in $1988[4,5]$ showed that the phase diagram for RADA is asymmetric. Two years later this result was confirmed by Kim and Kwun [7]. The dielectric dispersion of $\varepsilon^{\prime}$ nd $\varepsilon^{\prime \prime}$ in the paraelectric-proton glass transition, described by the Vogel-Fulcher expression, was revealed. Further dielectric studies led to the discovery of the coexistence of paraelectric/proton glass and long-range ordered phases. Analysis of the temperature dependence of $\varepsilon_{a}^{\prime \prime}$ in deuterated DRADA $x=0.39$ exposed the coexistence of antiferroelectric and glass phases. Two different relaxation mechanisms are involved: the Arrhenius type and the Vogel-Fulcher kind of relaxation. Thus, the phase coexistence for a mixed RADA crystal was confirmed.

\section{References}

1. Courtens E. Competing structural orderings and transitions to glass in mixed crystals of $\mathrm{Rb}_{1-x}\left(\mathrm{NH}_{4}\right)_{x} \mathrm{H}_{2} \mathrm{PO}_{4}$. // J. Phys. Lett. (Paris), 1982, vol. 43, p. L-199-L-204 .

2. Courtens E. Mixed crystals of the $\mathrm{KH}_{2} \mathrm{PO}_{4}$ family. // Ferroelectrics, 1987, vol. 72, p. 229-244.

3. Trybuła Z., Stankowski J., Blinc R. Proton glassy state of $\mathrm{Rb}_{1-x}\left(\mathrm{NH}_{4}\right)_{x} \mathrm{H}_{2} \mathrm{AsO}_{4}$. // Ferroelectrics Lett., 1986, vol. 6, p. 57-60.

4. Trybuła Z., Stankowski J., Szczepań ska L., Blinc R., Weiss Al., Dalal N.S. Proton glass state in $\mathrm{Rb}_{1-x}\left(\mathrm{NH}_{4}\right)_{x} \mathrm{H}_{2} \mathrm{AsO}_{4}$. // Ferroelectrics, 1988, vol. 79, p. 335-338.

5. Trybuła Z., Stankowski J., Szczepań ska L., Blinc R., Weiss Al., Dalal N.S. Proton glass state in $\mathrm{Rb}_{1-x}\left(\mathrm{NH}_{4}\right)_{x} \mathrm{H}_{2} \mathrm{AsO}_{4}$. // Physica B, 1988, vol. 153, p. 143-146.

6. Trybuła Z., Schmidt V.H., Drumheller J.E., He D., Li Z. Dielectric measurements of the proton-glass state in $\mathrm{Rb}_{0.65}\left(\mathrm{NH}_{4}\right)_{0.35} \mathrm{H}_{2} \mathrm{AsO}_{4}$. // Phys. Rev. B, 1989, vol. 40, No 7, p. 5289-5291.

7. Kim S., Kwun S. Proton glassy behavior in $\mathrm{Rb}_{1-x}\left(\mathrm{NH}_{4}\right)_{x} \mathrm{H}_{2} \mathrm{AsO}_{4}$ mixed crystal. // Phys. Rev. B, 1990, vol. 42, No 1, p. 638-642.

8. Trybuła Z., Schmidt V.H., Drumheller J.E. Coexistence of proton-glass and ferroelectric order in $\mathrm{Rb}_{1-x}\left(\mathrm{NH}_{4}\right)_{x} \mathrm{H}_{2} \mathrm{AsO}_{4}$. // Phys. Rev. B, 1991, vol. 43, No 1, p. 1287-1289. 
9. Eom J., Yoon J., Kwun S. Proton glass with remaining ferroelectric order in $\mathrm{Rb}_{1-x}\left(\mathrm{NH}_{4}\right)_{x} \mathrm{H}_{2} \mathrm{AsO}_{4}$ mixed crystal. // Phys. Rev. B, 1991, vol. 44, No 6, p. 28262829.

10. Howell F.L., Pinto N.J., Schmidt V.H. Complex permittivity of the deuterated and undeuterated proton glass $\mathrm{Rb}_{1-x}\left(\mathrm{NH}_{4}\right)_{x} \mathrm{H}_{2} \mathrm{AsO}_{4}$. // Phys. Rev. B, 1992, vol. 46, No 21, p. 13762-13766.

11. Trybuła Z., Stankowski J.Sz., Łoś Sz. Intermediate phase between deuteron-glass and antiferroelectric order in the D-RADA system. // Physica B, 1993, vol. 191, p. 312-316.

12. Pinto N.J, Schmidt V.H. Spontaneous polarization in the deuterated and undeuterated proton glass $\mathrm{Rb}_{1-x}\left(\mathrm{NH}_{4}\right)_{x} \mathrm{H}_{2} \mathrm{AsO}_{4}$. // Ferroelectrics, 1993, vol. 141, p. 207-213.

13. Trybuła Z., Waplak S., Stankowski J., Łoś Sz., Schmidt V.H., Drumheller J.E. Dielectric and EPR measurements of the deutereted glass D-RADA $x=0.46$. // Ferroelectrics, 1994, vol. 156, p. 371-376.

14. Levstik A., Kutnjak Z., Filipič C., Pirc R. Phase diagram in the $\mathrm{Rb}_{1-x}\left(\mathrm{NH}_{4}\right)_{x} \mathrm{D}_{2} \mathrm{PO}_{4}$ and $\mathrm{Rb}_{1-x}\left(\mathrm{NH}_{4}\right)_{x} \mathrm{H}_{2} \mathrm{AsO}_{4}$ Systems. // Ferroelectrics, 1995, vol. 168, p. 17-24.

15. Cevc P., de Loose J.P., Mac B., Cambel G., Dalal N.S., Blinc R. $\mathrm{Tl}^{2+}$ EPR detection of glassy behavior in $\mathrm{ADP}_{x}-\mathrm{RDP}_{1-x}$ and deuteration effects in the phase diagram of $\mathrm{ADP}_{x}-\mathrm{RDP}_{1-x}$. // Ferroelectrics, 1990, vol. 106, p. 113-118.

16. Samara G.A., Schmidt V.H. Pressure Dependence of the coexistence of proton-glass and ferro-/antiferroelectric order in $\mathrm{Rb}_{1-x}\left(\mathrm{NH}_{4}\right)_{x} \mathrm{H}_{2} \mathrm{AsO}_{4}$. // Ferroelectrics, 1995, vol. 168 , p. 239-250.

17. Blinc R., Zeks B. Soft modes in ferroelectric and antiferroelectrics. North- Holland, 1974.

18. Lines M.E., Glass A.M. Principies and application of ferroelectrics and related material. Clarendon Press, Oxford, 1977.

19. Slater J.C. //J. Chem. Phys., 1941, vol. 9, p. 16.

20. Takagi Y. // J. Phys. Soc. Japan, 1948, vol. 3, p. 271.

21. Courtens E., Rosenbaum T.F., Nagler S.E., Horn P.M. Short-range ordering and freezing in a randomly mixed ferroelectric-antiferroelectric crystal. // Phys. Rev.B, 1984, vol. 29, No 1, p. 515-518.

22. Terauchi H. Dipole-Glass Phase in $\mathrm{Rb}_{1-x}\left(\mathrm{NH}_{4}\right)_{x} \mathrm{H}_{2} \mathrm{PO}_{4}$. // Ferroelectrics, 1985, vol. 64 , p. $87-96$.

23. Terauchi H., Fatamura T., Nishihata Y., Iida S. // J. Phys. Soc. Japan, 1984, vol. 53, p. 483.

24. Takashige M., Terauchi H., Miura Y., Hoshino S. A re-entrant glasslike phase in $\mathrm{Rb}_{1-x}\left(\mathrm{NH}_{4}\right)_{x} \mathrm{H}_{2} \mathrm{PO}_{4}$. // J. Phys. Soc. Japan, 1985, vol. 54, No 9, p. 3250-3253.

25. Brü ckner H.J., Courtens E., Unruh H.G. Dielectric relaxation of mixed crysta ls of $\mathrm{Rb}_{1-x}\left(\mathrm{NH}_{4}\right)_{x} \mathrm{H}_{2} \mathrm{PO}_{4}$ at microwave frequencies. // Z. Phys. B: Cond. Matt., 1988, vol. 73 , p. 337-342.

26. Blinc R., Ailion D. C., Günther B., Zumer S. Nuclear magnetic resonance in random fields: cluster formation and local dynamics of deuteron glass. // Phys. Rev. Lett., 1986, vol. 57, p. 2826-2829.

27. He P. Dielectric dispersion in $\mathrm{Rb}_{1-x}\left[\mathrm{~N}\left(\mathrm{H}_{1-y} \mathrm{D}_{y}\right) 4\right]_{x}\left(\mathrm{H}_{1-y} \mathrm{D}_{y}\right)_{2} \mathrm{PO}_{4}$ mixed crystal system. // J. Phys. Soc. Japan, 1991, vol. 60, No 1, p. 313-323.

28. Miura Y., Matsunaga N., Orihara H., Mamiya T., Terauchi H. // Physica B, 1996, vol. 219-220, p. 279.

29. Nagata T., Iwata M., Orihara H., Ishibashi Y., Miura Y., Mamiya T., Terauchi H. 
Measurement of nonlinear dielectric constant in $\mathrm{Rb}_{1-x}\left(\mathrm{NH}_{4}\right)_{x} \mathrm{H}_{2} \mathrm{PO}_{4}$ mixed crystals. // J. Phys. Soc. Japan, 1997, vol. 66, No 5, p. 1503-1507.

30. Bärtschi P., Matthias W., Merz W., Scherrer P. // Helv. Phys. Acta, 1945, vol. 18, p. 240.

31. Busch G. // Helv. Phys. Acta, 1936, vol. 10, p. 261.

32. Matthias B., Merz W., Scherrer P. // Helv. Phys. Acta, 1947, vol. 20, p. 273.

33. Trybuia Z., Stankowski J., Gierszal H. Low-temperature x-band microwave dielectrometer. // Nauch. Apparat. ( Sci. Instrumentation ), 1988, vol. 3, No 2, p. 87-93.

34. Stankowski J., Trybuła Z. Proton glass - new state in solids. Postępy Fizyki Molekularnej (Molecular Physics Reports), 1990, vol. 3, p. 87-109 (in Polish).

35. Trybuła Z., Schmidt V.H., Drumheller J.E., Blinc R. Proton-glass state in $\mathrm{K}_{0.60}\left(\mathrm{NH}_{4}\right)_{0.40} \mathrm{H}_{2} \mathrm{AsO}_{4}$ detected by dielectric measurements. // Phys. Rev. B, 1990, vol. 42, No 10, p. 6733-6735.

36. Courtnes E. Vogel-Fulcher scaling of the susceptibility in a mixed-crystal proton glass. // Phys. Rev. Lett., 1984, vol. 52, No 1, p. 69-72.

37. Trybuła Z. // (private information).

38. Trybuła Z., Loś. Sz., Tu C.S., Schmidt V.H. The ferroelectric and proton glass coexistence region in $\mathrm{K}_{0.77}\left(\mathrm{NH}_{4}\right)_{0.23} \mathrm{H}_{2} \mathrm{AsO}_{4}$ detected by complex permittivity measurements. // J. Phys. Cond. Matt., 1995, vol. 7, p. 947-957.

39. Gridnev S.A., Korotkov L.N., Shuvalov L.A., Fedosyuk R.M. Phase coexistence in mixed $\mathrm{K}_{1-x}\left(\mathrm{NH}_{4}\right)_{x} \mathrm{H}_{2} \mathrm{PO}_{4}$ crystals. // Ferroelectrics, 1996, vol. 175, p. 107-110.

40. Landolt-Börnstein, New Series vol. 16. Ferroelectrics and related substances. K.H.Hellwege, A.M.Hellwege. Springer-Verlag, Berlin, 1982.

41. Presented in this paper in figure 21 from data by Z. Trybuła.

42. Hutton S.L., Fehst I., Bohmer R., Braune M., Mertz B., Lunkenheimer P., Loidl A. Proton glassy behavior and hopping conductivity in solid solutions of antiferroelectric betaine phosphate and ferroelectric betaine phosphite. // Phys. Rev. Lett., 1991, vol. 66, No 15, p. 1990-1993.

43. Angell C.A. // J. Phys. Chem. Solids, 1988, vol. 49, p. 863.

44. Pinto N.J., Ravindran K., Schmidt V.H. Field-heated, field-cooled, and zero-fieldheated static permittivity of deuteron glass $\mathrm{K}_{1-x}\left(\mathrm{NH}_{4}\right)_{x} \mathrm{H}_{2} \mathrm{AsO}_{4}$. // Phys. Rev. B, 1993, vol. 48, No 5, p. 3090-3094.

45. Samara G.A., Schmidt V.H. Pressure dependence of proton glass freezing in $\mathrm{Rb}_{1-x}\left(\mathrm{NH}_{4}\right)_{x} \mathrm{H}_{2} \mathrm{PO}_{4}$. // Phys. Rev. B, 1986, vol. 34, No 3, p. 2035-2037.

46. Samara G.A., Terauchi H. Pressure-induced suppresion of the proton-glass phase and isotope effects in $\mathrm{Rb}_{1-x}\left(\mathrm{NH}_{4}\right)_{x} \mathrm{H}_{2} \mathrm{PO}_{4}$. // Phys. Rev. Lett., 1987, vol. 59, No 3, p. 347350 .

47. Levstik A., Filipič C., Kutnjak Z., Levstik I., Pirc R., Tadię B., Blinc, R. Field-Cooled and Zero-Field cooled dielectric susceptibility in deuteron glasses. // Phys. Rev. Lett., 1991, vol. 66, No 18, p. 2368-2371.

48. Sherrington D., Kirpatrick S. // Phys. Rev. Lett., 1975, vol. 35, p. 1792.

49. Pirc R., Tadič B., Bilnc R. // Phys. Rev. B, 1987, vol. 36, p. 8607.

50. Blinc R., Dolinšek J., Pirc R., Tadič B., Zalar B., Kind R., Liechti O. Localpolarization distribution in deuteron glasses. // Phys. Rev. Lett., 1989, No 20, vol. 63, p. 2248-2251.

51. Prelovšek P., Blinc R. Spin glass phase in mixed ferroelectric-antiferroelectric hydrogen bonded systems. // J. Phys. C: Solid State Phys., 1982, vol. 15, p. L985-L990. 
52. Matsushita E., Matsubara T. Cluster theory of glass transition in $\mathrm{Rb}_{1-x}\left(\mathrm{NH}_{4}\right)_{x} \mathrm{H}_{2} \mathrm{PO}_{4}$. // J. Phys. Soc. Japan, vol. 54, No 3, p. 1161-1167.

\section{Співіснування параелектричного/протонне скло та сегнетоелектричного (антисегнетоелектричного) впорядкування в кристалах $\mathrm{Rb}_{1-x}\left(\mathrm{NH}_{4}\right)_{x} \mathrm{H}_{2} \mathrm{AsO}_{4}$}

\section{3.Трибула, Я.Станковскі}

Інститут молекулярної фізики ПАН,

Польща, 60-179, Познань, вул. Смолуховського, 17

Отримано 17 червня 1998 р.

Ця стаття $є$ оглядом результатів діелектричного вивчення стану протонного скла у змішаних кристалах $\mathrm{Rb}_{1-x}\left(\mathrm{NH}_{4}\right)_{x} \mathrm{H}_{2} \mathrm{AsO}_{4}$ (RADA). Співіснування впорядкувань параелектричного/протонне скло та сегнетоелектричного чи антисегнетоелектричного, підтверджене іншими дослідженнями, детально обговорюється. Фазова діаграма RADA $€$ асиметричною. Стан протонного скла існує для концентрації аміаку в межах $0,1<x<0,5$. Представлена фазова діаграма дейтерованого DRADA. Область стану протонного скла в DRADA $(0,2<x<$ $0,35)$ є вужчою, ніж у недейтерованому RADA. Представлені ефекти впливу гідростатичного тиску на діелектричні властивості кристалу в стані протонного скла. Температура склування $T_{g}$ спадає з тиском і за оцінками прямує до нуля при 5 кбар. Низькотемпературна поведінка є все ще дискусійною, оскільки немає експериментально підтвердженої $T_{g}(p)$ залежності нижче $4 \mathrm{~K}$ для систем протонного скла

Ключові слова: доменна структура, сегнетоелектрики

PACS: $77.22 \mathrm{ch}, 77.22 \mathrm{gm}, 64.70-p, 74.84-\mathrm{s}$ 